\title{
Nuevas alternativas a los métodos industrialmente implantados para el sellado del aluminio anodizado ${ }^{(\bullet)}$
}

\author{
A. Bautista ${ }^{(*)}$, R. Lizarbe ${ }^{(\dagger)(*)}$, E. Otero ${ }^{(*)}$, V. López $^{(*)}$ y J.A. González ${ }^{(*)}$
}

Resumen Los resultados presentados en este trabajo permiten diseñar nuevos procesos de sellado industrial de los óxidos anódicos de aluminio. Gracias a la combinación del poder catalítico de la trietanolamina (TEA) y los efectos beneficiosos del envejecimiento, los procesos propuestos ofrecen importantes ventajas si se comparan con el sellado en agua a ebullición y el sellado en frío, que son los métodos de sellado más utilizados en la actualidad.

Palabras clave: Aluminio anodizado. Sellado. Trietanolamina. Envejecimiento.

\section{New alternatives to the industrially introduced methods for the sealing of anodized aluminium}

\begin{abstract}
The results shown in this paper allow the design of new processes for the industrial sealing of anodic oxides of aluminium. Thanks to the combination of the cathalitic power of triethanolamine (TEA) and the beneficial effects of ageing, the proposed processes offer considerable advantages compared with boiling water sealing and cold sealing, which are the most utilized sealing methods nowadays.
\end{abstract}

Keywords: Anodized aluminium. Sealing. Triethanolamine. Ageing.

\section{INTRODUCCIÓN}

Cuando una superficie de aluminio recién formada se expone a la atmósfera, se recubre en fracciones de segundo de una capa muy delgada de óxido de aluminio o alúmina $\left(\mathrm{Al}_{2} \mathrm{O}_{3}\right)$ que pasiva el metal y evita su deterioro espontáneo. El pequeño espesor de esta capa de óxido (2-3 $\mathrm{nm}$ ) hace que, en determinados ambientes, sea frecuente su ruptura y se inicie la corrosión del aluminio, normalmente por picaduras.

La anodización del aluminio (1) es un procedimiento industrial que tiene por objeto aumentar en unos 4 órdenes de magnitud el espesor de la capa natural de alúmina, para disminuir la susceptibilidad a la corrosión del metal. Los óxidos obtenidos artificialmente son extremadamente porosos y tien-

$(\bullet \quad$ Trabajo recibido el día 9 de junio de 1999 y aceptado en su forma final el día 1 de julio de 1999.

(*) Centro Nacional de Investigaciones Metalúrgicas, CENIM (CSIC). Av. Gregorio del Amo, 8. 28040Madrid (España). den a absorber suciedad e iones agresivos cuando estos se encuentran presentes en la atmósfera, por lo que, tras el anodizado, se suele someter al aluminio a una etapa de sellado.

El sellado consiste, tradicionalmente, en la inmersión de las piezas anodizadas en agua destilada o desionizada a temperatura de ebullición, lo que provoca la hidratación de la alúmina y la formación de compuestos de mayor volumen específico, que precipitan en la boca de los poros, eliminando o reduciendo drásticamente la porosidad de las capas (2). También existen otros métodos de sellado con importancia industrial que sustituyen el agua a ebullición por una disolución en la cual se hallan presentes iones que también precipitan, junto con la alúmina hidratada, en la boca de los poros, permitiendo reducir el tiempo y/o la temperatura de sellado (3). El más conocido de todos estos procedimientos es el sellado en frío (4).

El gran consumo energético que implica el sellado en agua a ebullición y el elevado coste y los problemas tóxicos, e incluso alérgicos, a los que dan lugar las sales empleadas en los baños de sellado en 
frío, han impulsado la búsqueda de alternativas a los procesos actualmente implantados en la industria. Para ello se ha investigado la cinética de sellado en disoluciones de acetato sódico y de trietanolamina (TEA), aditivos orgánicos ya propuestos en la bibliografía como aceleradores del sellado (5-9), en condiciones hasta ahora nunca estudiadas. En este trabajo se presentan los resultados con una mayor implicación práctica de entre todos los obtenidos.

\section{EXPERIMENTAL}

Los estudios se realizaron sobre probetas de aluminio de pureza comercial 99,5\%, sin anodizar y anodizadas en disoluciones de ácido sulfúrico del $18 \%$ en masa, a $20^{\circ} \mathrm{C}$ y $1,5 \mathrm{~A} / \mathrm{dm}^{2}$ durante tiempos suficientes para generar capas de óxido de, normalmente, $20 \mu \mathrm{m}$ de espesor y, en algún caso, de 6 y $16 \mu \mathrm{m}$. Las probetas anodizadas se estudiaron sin sellar, tras ser selladas durante distintos tiempos en agua desionizada, en disoluciones de acetato sódico de $0,5 \mathrm{~g} / \mathrm{l}$ de ion acetato y en disoluciones de $2 \mathrm{~cm}^{3} / 1$ de TEA, a temperaturas comprendidas entre los 50 y los $100^{\circ} \mathrm{C}$, y después de un sellado en frío, a 25 $30{ }^{\circ} \mathrm{C}$, durante 20 minutos, en disoluciones de 1,63 $\mathrm{g} / \mathrm{l}$ de iones $\mathrm{Ni}^{2+}$ y $0,7 \mathrm{~g} / \mathrm{l}$ de iones $\mathrm{F}^{-}$, preparadas a partir de $\mathrm{NiF}_{2}$ y $\mathrm{NiAc}_{2}$. Muchas de estas probetas fueron envejecidas posteriormente en atmósferas naturales o artificiales a muy diferentes temperaturas y humedades relativas (HR), para profundizar en el conocimiento de las transformaciones espontáneas de los óxidos anódicos.

Como métodos de estudio se utilizaron la inspección visual y técnicas gravimétricas y se recurrió a los ensayos normalizados para el control de la calidad del sellado: el ensayo de absorción de la gota de colorante (10), la medida de la admitancia a $1 \mathrm{kHz}(11)$ y el ensayo de inercia a la disolución ácida en medio fosfocrómico (12).

\section{RESULTADOS}

El aluminio desnudo presenta problemas de corrosión localizada en atmósferas naturales cuya contaminación por $\mathrm{Cl}^{-}$supera los $30 \mathrm{mg} / \mathrm{m}^{2}$. día, tal y como se aprecia mediante la inspección visual de probetas de este material expuestas en diferentes estaciones de ensayo del proyecto PATINA y como se refleja en las pérdidas de peso que experimentan estas probetas tras 1 año de exposición (Fig. 1). El anodizado y sellado del aluminio han sido capaces de inhibir el ataque por picaduras durante este mismo periodo en la atmósfera, incluso en los ambientes más agresivos. Tal y como se observa en la figura 1, las probetas anodizadas y selladas de

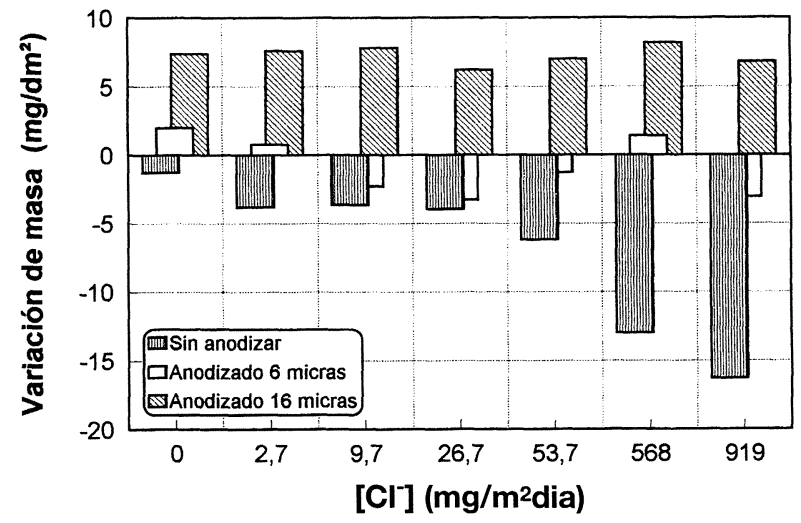

FIG. 1.-Variaciones de masa experimentadas por probetas de aluminio desnudo y aluminio anodizado y sellado durante 60 min en agua a ebullición, tras 1 año de exposición en atmósferas naturales con muy diferente grado de contaminación por cloruros.

FIG. 1.- Mass changes undergone by bare aluminium and anodized aluminium specimens sealed 60 min in boiling water, after 1 year of exposition in natural atmospheres with very different levels of chloride contamination.

menor espesor no sufren variaciones significativas de peso durante la exposición, mientras que aquellas con capas de óxido más gruesas experimentan, durante el primer año, una ganancia de masa que resulta ser independiente de las características de la atmósfera a la que están expuestas.

Los recubrimientos sellados expuestos a la atmósfera experimentan una lenta pero continua pérdida de peso (Fig. 2) que refleja la disminución uniforme de su espesor, que tiene lugar debido a la acción erosiva de la atmósfera. Cuando las capas

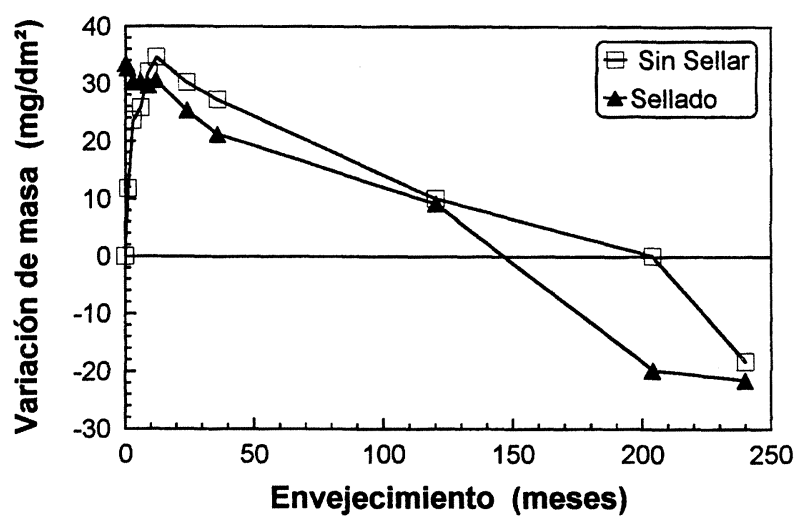

FIG. 2.- Variaciones de masa experimentadas por recubrimientos anódicos de $20 \mu \mathrm{m}$ de espesor, sin sellar y sellados en agua a ebullición, durante 20 años de exposición a la atmósfera de Madrid.

FIG. 2.- Mass changes undergone by $20 \mu \mathrm{m}$ anodic coatings, unsealed and sealed in boiling water, during 20 years of exposition to the Madrid atmosphere. 
anódicas se exponen sin sellar (Fig. 2), se observa una ganancia de masa, durante las primeras semanas. Al cabo de un cierto tiempo, el peso de estos recubrimientos iguala al que hubieran logrado óxidos de las mismas características tras una inmersión lo suficientemente prolongada en el baño de sellado y empiezan a predominar también los fenómenos de desgaste. El desgaste de los anodizados es tan lento, en atmósferas de agresividad moderada como la de Madrid, que, en el peor de los casos, la pérdida de

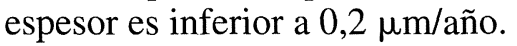

La adición de iones acetato al baño de sellado reduce el tiempo de inmersión mínimo, requerido por el método tradicional en agua a temperatura de ebullición, para superar los ensayos normalizados. Los 10 min de sellado, necesarios para disminuir la intensidad de la gota de colorante por debajo del límite de "2" exigido en la norma, se transforman en $\tan$ solo $5 \mathrm{~min}$ en disoluciones de acetato (Fig. $3 a)$, y los 12-15 min, requeridos para reducir las pérdidas de peso en ácido fosfocrómico por debajo de los $30 \mathrm{mg} / \mathrm{dm}^{2}$, se reducen a la tercera parte gracias a este aditivo (Fig. 3b).
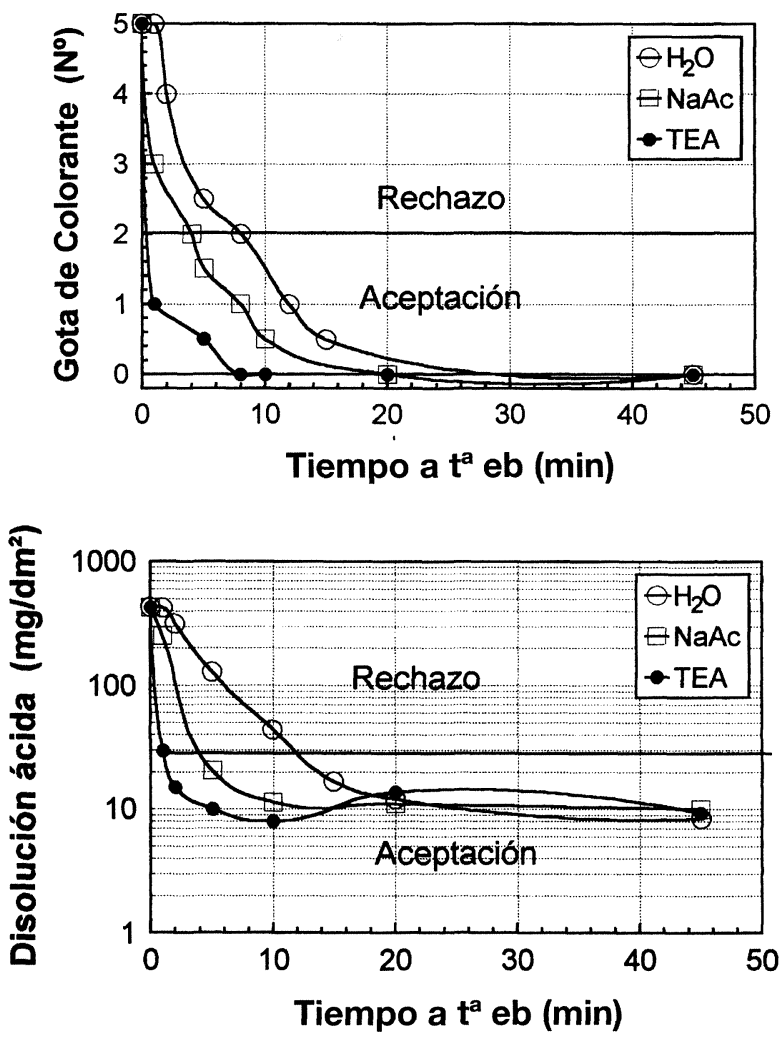

FIg. 3.- Evolución de los resultados de los ensayos de la gota de colorante y la inercia a la disolución ácida con el tiempo de sellado en distintos medios, a temperatura de ebullición, de los óxidos anódicos.

FIG. 3.- Evolution of the results of the dye spot and the acid dissolutions test with the sealing time in different media, at the boiling temperature, of the anodic oxides.
El efecto catalítico de la TEA sobre el proceso de sellado es todavía mucho mayor que el de los iones acetato. En solamente $1 \mathrm{~min}$ a temperatura de ebullición, la calidad de los sellados obtenidos en disoluciones de TEA es suficiente para superar los ensayos de la gota de colorante y la disolución ácida (Fig. 3). El efecto positivo de este compuesto orgánico también se observa claramente mediante el ensayo de admitancia (Fig. 4).

A pesar de que la velocidad de las reacciones de hidratación de la alúmina disminuye siempre, de forma muy acusada, con la temperatura (13), el efecto catalizador de los aditivos orgánicos empleados es relativamente mayor cuanto menor es la temperatura de sellado. En la figura 4 se observa, por ejemplo, que si la TEA reduce a la mitad el tiempo de inmersión necesario a temperatura de ebullición, para que la admitancia a $1 \mathrm{kHz}$ del recubrimiento disminuya por debajo de los $20\left(\mathrm{~S}\right.$ a $50^{\circ} \mathrm{C}$, este aditivo puede permitir una reducción de $12-13$ veces el tiempo de sellado requerido en agua desionizada.

A cualquier temperatura, los requisitos del ensayo de la admitancia exigen inmersiones más prolongadas que los de la gota de colorante o la disolución ácida para poder cumplirse (figs. 3-5). Resulta interesante destacar, por ejemplo, que, aún recurriendo a los efectos beneficiosos de la TEA, la obtención de sellados que superen el ensayo de admitancia en tiempos compatibles con la práctica industrial (inferiores a 1 hora) implica temperaturas del baño de $90{ }^{\circ} \mathrm{C}$ o superiores, mientras que, según el ensayo de la disolución ácida, se pueden realizar sellados en disoluciones de TEA a $80^{\circ} \mathrm{C}$ en tan sólo

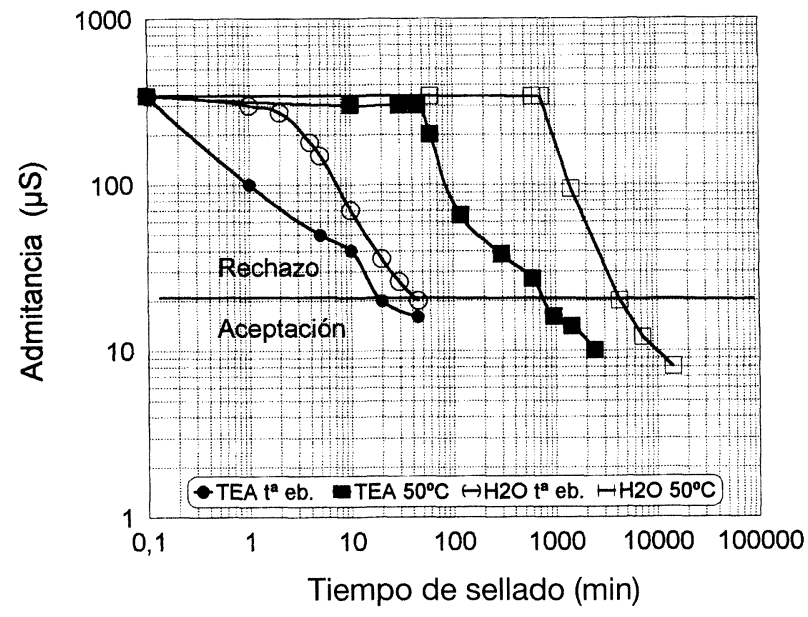

Fig. 4.- Evolución de la admitancia a $1 \mathrm{kHz}$ con el tiempo de inmersión de las capas anódicas en agua desionizada y en disoluciones de TEA a temperatura de ebullición y a $50{ }^{\circ} \mathrm{C}$.

FIG. 4.- Evolution of the $1 \mathrm{kHz}$ admittance with the immersion time of the anodic layers in de-ionized water and TEA solutions at boiling temperature and at $50^{\circ} \mathrm{C}$. 
8-10 min y se puede reducir la temperatura del baño hasta unos $65^{\circ} \mathrm{C}$, si se está dispuesto a prolongar $1 \mathrm{~h}$ el proceso (Fig. 5).

La exposición a la atmósfera de recubrimientos sellados durante corto tiempo en diferentes medios, de forma que inicialmente no cumplen en ningún caso las exigencias del ensayo de admitancia, da lugar a un aumento continuo y espontáneo del grado de sellado y al cumplimiento, al cabo de un cierto tiempo, de todas las condiciones exigidas en la normativa (Fig. 6). Por ejemplo, sellados de 8 min a temperatura de ebullición, realizados tanto mediante el método tradicional como en presencia de aditivos orgánicos, presentan, tras 6 meses en la atmósfera de Madrid, una admitancia claramente inferior a $20 \mu \mathrm{S}$.

La velocidad de las transformaciones beneficiosas que tienen lugar espontáneamente depende, en gran medida, de las condiciones climatológicas del ambiente al cual se expongan las capas anódicas. La temperatura acelera de forma muy pronunciada la hidratación de la alúmina. Un aumento de este parámetro reduce espectacularmente el tiempo necesario para que un sellado incompleto se transforme en uno de calidad (Fig. 7). La humedad también puede influir, especialmente si el grado de sellado inicial de los poros es muy bajo, pero como se observa en la figura 8 , la capacidad higroscópica del óxido es tal, que se requieren concentraciones muy bajas de agua en la atmósfera, imposibles de encontrar en ambientes naturales, para ralentizar significativamente el proceso de obturación de los poros.

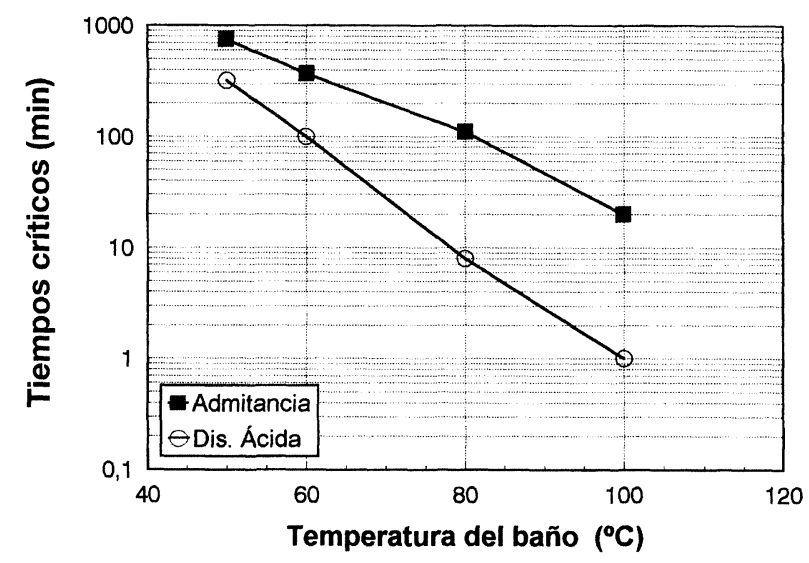

FIG. 5.- Relación entre el tiempo de inmersión mínimo para cumplir los requisitos de los ensayos normalizados de la disolución ácida y la admitancia a $1 \mathrm{kHz}$ y la temperatura de la disolución de TEA donde se lleva a cabo el sellado.

FIG. 5.- Relationship between the minimum immersion time for fulfilling the requirements of the acid solution and $1 \mathrm{kHz}$ admittance normalized tests and the temperature of the TEA solution where sealing is being carried out.

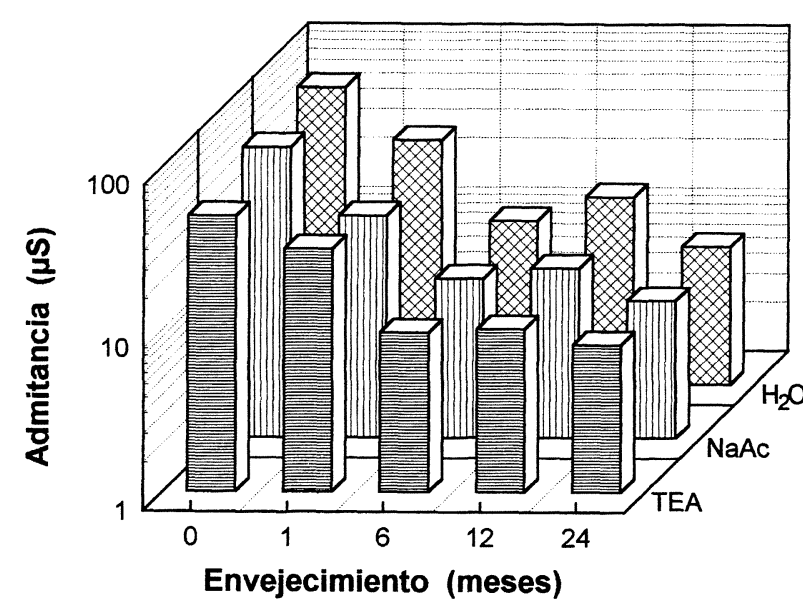

Fig. 6.- Evolución, con el tiempo de exposición en la atmósfera de Madrid, de la admitancia a $1 \mathrm{kHz}$ de capas anódicas selladas durante 8 minutos en diferentes medios a temperatura de ebullición.

FIG. 6.- Evolution, with the exposure time to the Madrid atmosphere, of the $1 \mathrm{kHz}$ admittance of the anodic layers sealed for 8 minutes in different media at boiling temperature.

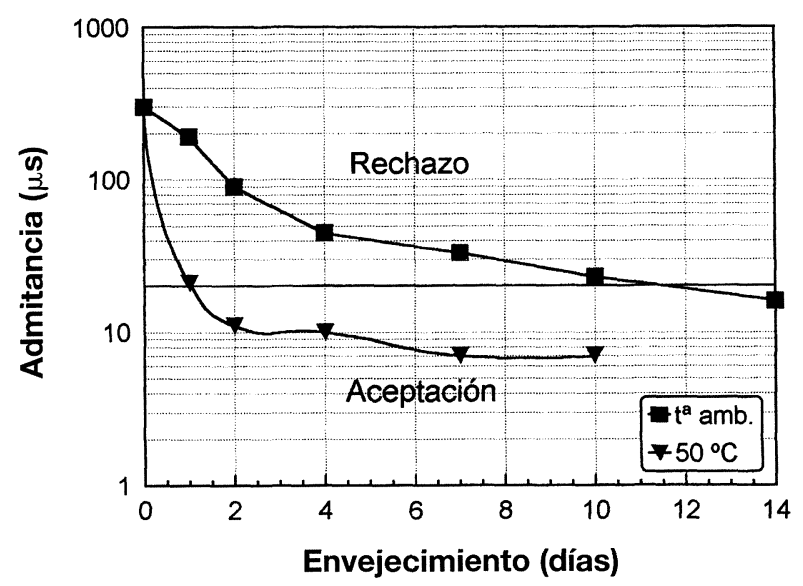

FIg. 7.- Evolución, con el tiempo de exposición a una atmósfera de alta HR, a temperatura ambiente y a $50^{\circ} \mathrm{C}$, de la admitancia a $1 \mathrm{kHz}$ de capas anódicas selladas durante $30 \mathrm{~min}$ en disoluciones de TEA a $50{ }^{\circ} \mathrm{C}$.

FIG. 7.-Evolution, with the exposure time at a high $\mathrm{RH}$ atmosphere, at room temperature and at $50^{\circ} \mathrm{C}$, of the $1 \mathrm{kHz}$ admittance of anodic layers sealed for $30 \mathrm{~min}$ in TEA solutions at $50^{\circ} \mathrm{C}$.

Aunque un envejecimiento de recubrimientos sin sellar en la atmósfera de Madrid dé lugar a altas calidades de sellado y ninguna de las muchas probetas expuestas haya presentado problemas de corrosión, sí se puede observar un importante deterioro estético de las mismas, debido a la suciedad absorbida dentro de los poros antes de que se obturen (Fig. 9). 


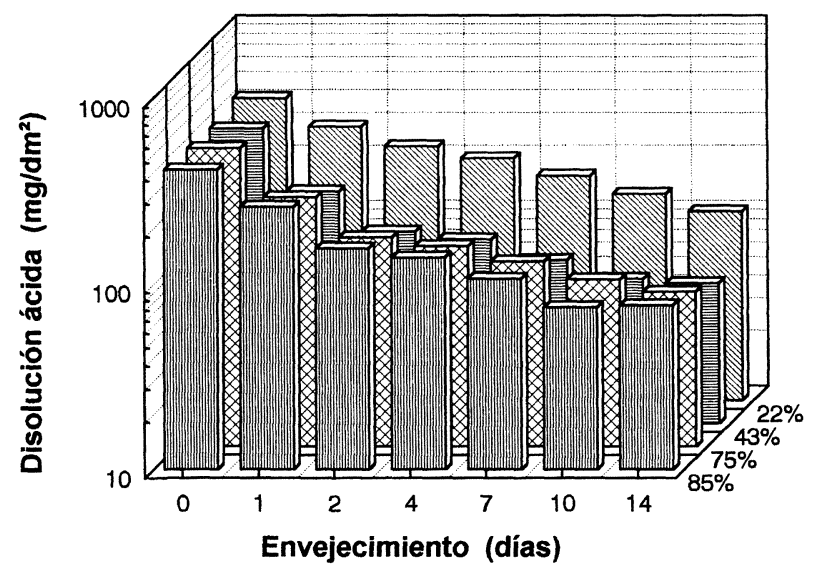

FIG. 8.- Evolución, con el tiempo de exposición a atmósferas artificiales de muy diferente HR, a temperatura ambiente, de las pérdidas de peso experimentadas en el ensayo de inercia a la disolución ácida por probetas selladas durante 30 min en TEA a $50^{\circ} \mathrm{C}$.

FIG. 8.- Evolution, with the exposure time at a high $\mathrm{RH}$ atmosphere, at room temperature, of the mass losses undergone by anodic layers sealed for $30 \mathrm{~min}$ in TEA solutions at $50^{\circ} \mathrm{C}$ during the acid solution test.

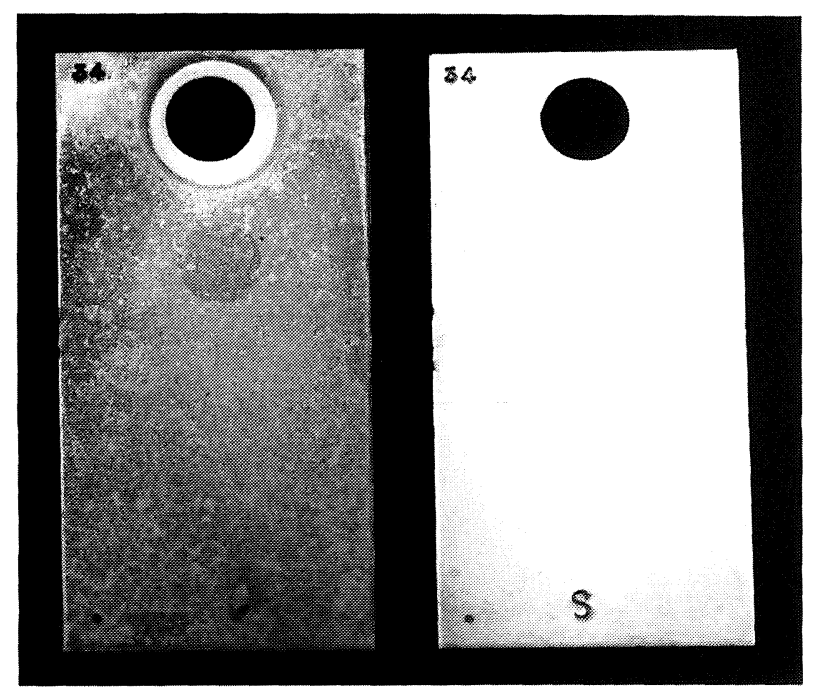

FIG. 9.- Aspecto de una probeta de aluminio anodizado sin sellar y otra sellada tras 3 años de exposición en la atmósfera de Madrid.

FIG. 9.- Appearance of unsealed and sealed anodized aluminium specimens after 3 years of exposure to the Madrid atmosphere.

Los óxidos sellados en frío no cumplen ninguno de los requisitos de calidad exigidos por los ensayos normalizados inmediatamente después de ser extraídos del baño de sellado, pero sí lo hacen tras un periodo de envejecimiento que, a temperatura ambiente, es inferior a $24 \mathrm{~h}$ para los ensayos de la gota de colorante y la disolución ácida (Fig. 10) y

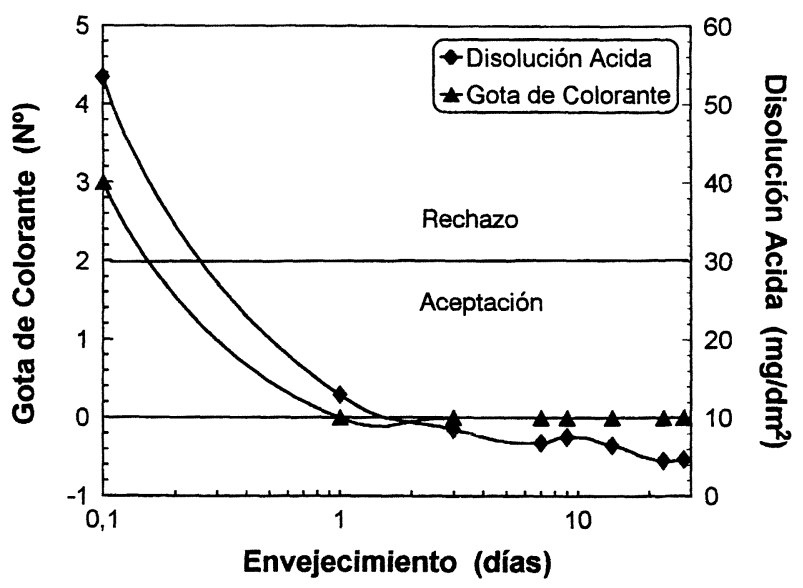

FIG. 10.- Evolución, con el tiempo de exposición a una atmósfera de alta HR a temperatura ambiente, de los resultados de los ensayos de la gota de colorante y la inercia a la disolución ácida para recubrimientos sellados en frío.

FIG. 10.-Evolution, with the exposure time to a high $R H$ atmosphere at room temperature, of the results of the dye spot and acid solution tests for cold-sealed coatings.

que tiene que prolongarse hasta casi 3 días para el ensayo de la admitancia (Fig. 11). Como en otros casos, la velocidad de las transformaciones que tienen lugar en este tipo de sellados aumenta con la temperatura y, cuando el envejecimiento ocurre a $50^{\circ} \mathrm{C}$, el tiempo necesario para superar el ensayo de admitancia se reduce a $4 \mathrm{~h}$.

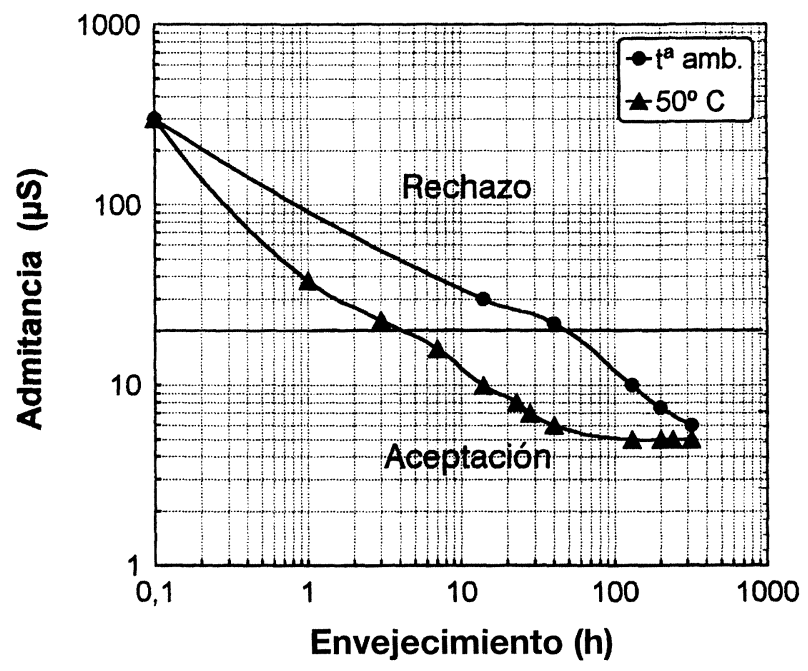

Fig. 11.- Evolución, con el tiempo de exposición a una atmósfera de alta HR a temperatura ambiente y a $50{ }^{\circ} \mathrm{C}$, de la admitancia a $1 \mathrm{kHz}$ de óxidos sellados en frío.

FIG. 11.- Evolution, with the exposure time to a high RH atmosphere at room temperature and at $50^{\circ} \mathrm{C}$, of $1 \mathrm{kHz}$ admittance of cold-sealed coatings. 


\section{DISCUSIÓN}

\subsection{Capacidad protectora de los recubrimientos anódicos}

El anodizado y sellado del aluminio es capaz de inhibir la aparición de los problemas de corrosión localizada que afectan al aluminio desnudo en atmósferas muy contaminadas (Fig. 1). Para sellados de igual duración, esta capacidad protectora parece ser independiente del espesor de la capa anódica, al menos durante el primer año de exposición. Por otro lado, las velocidades de desgaste registradas en atmósferas de agresividad moderada (Fig. 2) permiten estimar, para capas de $20 \mu \mathrm{m}$ habituales en aplicaciones arquitectónicas, durabilidades del orden de los 100 años o superiores para los anodizados. Aunque estas velocidades de desgaste son muy bajas, es probable que, para vidas en servicio muy prolongadas, la erosión de las capas de alúmina más delgadas pudiera llegar a introducir diferencias en el comportamiento frente a la corrosión de anodizados de distinto espesor.

Las ganancias de masa que experimentan las capas anódicas cuando son expuestas a la atmósfera (Figs. 1 y 2) no están relacionadas con procesos de corrosión, sino con fenómenos de absorción de agua dentro de los poros y dependen, para periodos de exposición suficientemente prolongados, de las características del sellado y del volumen de porosidad que presenten inicialmente los óxidos (14 y 15).

\subsection{Posibilidad de sellar de forma más económica utilizando aditivos orgánicos}

Considerando los resultados del ensayo de admitancia que, como ya se ha comentado, es el más exigente de todos, se observa que se puede reducir a la mitad el tiempo requerido para el sellado hidrotérmico tradicional, sin que esto suponga ninguna alteración en el grado de hidratación de los recubrimientos obtenido tras el proceso industrial, simplemente añadiendo TEA al baño de sellado (Figs. 3 y 4). Esto permitiría tratar la producción de 2 baños de anodizado en un único baño de sellado, lo que supondría un sustancial ahorro de espacio y, lo que es más importante, de energía. (Si se usaran, como criterio para conseguir un sellado aceptable, los resultados proporcionados por otros ensayos normalizados se podría reducir mucho más el tiempo de sellado, tal y como se explica en el siguiente apartado).

El empleo de TEA también permite obtener sellados que cumplen con todos los requisitos de calidad, es decir, que superan el ensayo de admitan- cia a temperaturas inferiores a la de ebullición y en tiempos compatibles con la práctica industrial. A pesar de que la velocidad de sellado (que se puede considerar inversamente proporcional al tiempo crítico necesario para superar los ensayos normalizados) decrece exponencialmente cuando baja la temperatura del baño (Fig. 5), el poder catalizador de la TEA es tal que puede permitir reducir la temperatura de la cuba de sellado unos $10{ }^{\circ} \mathrm{C}$ sin que esto retrase el ritmo de la cadena de producción, pues el tiempo invertido en el sellado sería similar al requerido por la anodización. Aunque esta disminución de temperatura no es, en sí misma, muy espectacular, sí que podría tener interesantes consecuencias económicas. El importante coste del sellado hidrotérmico se debe, fundamentalmente, al gran consumo energético que implica el calor de cambio de estado del agua. Si se reducen las pérdidas por evaporación, no sólo se disminuyen los problemas e incomodidades que acarrean las condensaciones sino que se logra un importante ahorro de energía. La disminución de la temperatura de sellado en disoluciones de TEA puede ser todavía muchísimo mayor si se tienen en cuenta los rápidos efectos beneficiosos del envejecimiento de sellados inicialmente incompletos.

\subsection{Aplicación práctica de los efectos beneficiosos del envejecimiento}

Los sellados insuficientes reducen sustancialmente la capacidad absorbente de los anodizados, de manera que no tiene lugar la absorción de suciedad dentro de los poros, que ocurre cuando se exponen a la atmósfera óxidos sin sellar (Fig. 9), y la conservación del aspecto estético es tan eficaz como la que proporcionan los sellados que cumplen todas las exigencias de las normas inmediatamente después de la inmersión. La superación de los ensayos de la gota de colorante y la disolución ácida garantizan que la boca de los poros se encuentra totalmente obturada (15) y que no sólo la suciedad, sino tampoco los iones agresivos que pudieran estar presentes en ambientes más corrosivos, son capaces de penetrar en el interior de los poros ni, por supuesto, alcanzar al metal base. Además, el proceso de hidratación de la alúmina continúa espontáneamente en la atmósfera tras el sellado, dando lugar a una mejora de su calidad (Fig. 6). Este aumento del grado de sellado parece ser una característica de los sellados incompletos, siempre que se supere un mínimo de HR no bien establecido hasta el momento, pero que, evidentemente, se sobrepasa en cualquier ambiente natural en el que puedan ser expuestas las piezas anodizadas (Fig. 8). Por tanto, es simplemente cuestión de tiempo que un sellado que 
no cumple las exigencias del ensayo de admitancia, pero cuyos poros están lo suficientemente bloqueados para garantizar una buena resistencia a la corrosión, cumpla con todos los requisitos de la normativa.

La aceptación de esta evidencia experimental podría llevar a proponer la realización de sellados que, simplemente, cumplieran las exigencias del ensayo de la disolución ácida. Esto permitiría sellar, en disoluciones de TEA a temperatura de ebullición, en tiempos cortísimos (Fig. 3) o, lo que quizá resultara más interesante, reducir significativamente la temperatura de operación sin que ello significara un aumento del tiempo de inmersión. Los datos de la figura 5 pueden resultar muy útiles a la hora de diseñar procesos de sellado, jugando con el trinomio tiempo de sellado-temperatura-número de baños, para ajustar la velocidad del proceso a los diferentes ritmos de demanda que pueda presentar la cadena de producción.

Los efectos beneficiosos del envejecimiento también son muy acusados en los recubrimientos sellados en frío (Figs. 10 y 11), y la rápidez con que tiene lugar el bloqueo de la boca de los poros y el aumento de la calidad del sellado tras la inmersión parece sugerir, que la etapa de postsellado a mayor temperatura que muchas veces se lleva a cabo (16 y 17) y que encarece significativamente el proceso es, en realidad, innecesaria.

Aunque el sellado en frío permite realizar inmersiones a temperaturas aún más bajas y los óxidos envejecen más rápidamente que los sellados en TEA, el fluoruro de níquel presenta, sin embargo, las desventajas de ser un aditivo más caro y de requerir un control de los baños más complicado para el desarrollo óptimo del proceso (15). Esto hace interesante el diseño de nuevos procedimientos en dos etapas, la primera de las cuales consistiría en un presellado de corta duración a baja temperatura, en una disolución con catalizadores orgánicos, seguida de un periodo de envejecimiento en condiciones controladas. En estos casos, al igual que tras el sellado en frío, no se cumplirían, inmediatamente después de la inmersión, ninguno de los mínimos de calidad exigidos actualmente por la normativa, pero sí después de un corto periodo de postsellado o almacenamiento. Por ejemplo, un presellado en TEA a $50{ }^{\circ} \mathrm{C}$ necesita menos de dos semanas a temperatura ambiente para disminuir su admitancia por debajo de los $20 \mu \mathrm{S}$, y tan sólo $24 \mathrm{~h}$ si el envejecimiento tiene lugar a $50^{\circ} \mathrm{C}$ (Fig. 7).

\section{CONCLUSIONES}

El sellado industrial de las capas anódicas de aluminio se puede llevar a cabo de una forma económica- mente más ventajosa y sin que ello implique ningún riesgo en su comportamiento frente a la corrosión, si se tienen en cuenta las siguientes evidencias experimentales:

- El empleo de TEA acelera significativamente el sellado y permite reducir el tiempo de inmersión y/o la temperatura del baño.

- La obturación de los poros, es decir, la superación de los ensayos de la gota de colorante y la inercia a la disolución ácida, bastan para garantizar la resistencia a la corrosión y la conservación del aspecto estético de las piezas anodizadas.

- Los evidentes efectos beneficiosos del envejecimiento sobre sellados inicialmente incompletos, tanto realizados en frío como a temperaturas superiores, puede permitir acortar el tiempo de inmersión y/o disminuir la temperatura del baño de sellado.

\section{Agradecimientos}

Este trabajo, pretende, ante todo, ser un recuerdo agradecido al Dr. Rafael Lizarbe, por el regalo de su amistad y por su capacidad de servicio desinteresado a todo el que, relacionado con la anodización y sellado, acudía a él en busca de asesoramiento y consejo.

Trabajo financiado por la Comisión Interministerial de Ciencia y Tecnología a través del Proyecto MAT98-096-CO2-01.

\section{REFERENCIAS}

(1) Wernick, S., Pinner, R., y Sheasby, P.G. The Surface Treatment and Finishing of aluminium and its alloys, Vol. 1, $5^{\text {a }}$ ed., ASM International, Metals Park, Ohio, EE. UUU: $289-368$,

(2) Wefers, K., Aluminium, 49 (8), 1973: 553-561, 49 (9), 1973: 622-624.

(3) YAfFe, B., Met. Finish, 88 (5), 1990: 41-45

(4) Kalantary, M.R., Gabe, D.R., y Ross, D.H., Alum. Finish., 13 (4-5), 1993: 33-37.

(5) Manhart, J.H., y Cochran, W.C., Plating, 58 (3), 1971 : 219-224

(6) Lizarbe, R., y de la Orden, M.A., Rev. Metal. Madrid, 20 (1), 1984: 44-51.

(7) Lizarbe, R., Rev. Metal. Madrid, 24 (6), 1988: 402-410.

(8) Olin Mathieson Chemical Corp., Brit. Pat. 3,822,377 (1968). 
(9) Alcan Research and Development Ltd., European Pat. 0 005919 (1979) ; US Pat. 4,288,299 (1981).

(10) Norma UNE 38-017, "Evaluación de la calidad del sellado de la capa de óxido de aluminio anodizado Método de la gota de colorante”, Marzo 1982.

(11) Norma UNE 38-026 "Anodización del aluminio y sus aleacciones - Evaluación de la calidad del sellado de los recubrimientos anódicos de óxido por medida de la admitancia", Enero 1993.

(12) Norma UNE 38-016, "Evaluación de la calidad del sellado de la capa de óxido del aluminio anodizado Método de inercia a la disolución en medio acético" Octubre 1972.
(13) Bautista, A., Otero, E., Lopez, V., y Gonzalez, J.A., Plat. Surf. Finish., 85 (5), 1998: 110-114.

(14) Bautista, A., Morcillo, M., Gonzalez, J.A., Simancas, J., y Otero, E., Rev. Metal. Madrid, 34 (Núm. Extr., mayo), 1998: 32-36.

(15) Bautista, A., Tesis Doctoral, Fac. de CC. Químicas de la Universidad Complutense de Madrid, Madrid, 1998.

(16) Kalantary, M.R., Gabe, D.R, y Ross, D.H., Trans. Inst. Metal Finish., 70 (2), 1992: 62-67.

(17) Qualanod specification "for cold seal processing based on nickel fluoride”, 1990. 